\title{
Kulturwechsel und Erkrankung - von Unterschieden und Gemeinsamkeiten
}

\author{
Je nach kulturellem Hintergrund sind auch unterschiedliche Vorstellungen von \\ Gesundheit und Krankheit wirksam, was die medizinische Betreuung von Menschen \\ mit Migrationshintergrund anspruchsvoll, aber auch interessant macht.
}

\section{Lenka Svejda-Hirsch ${ }^{a}$,} Peter Streb ${ }^{b}$

a lic. phil., MAS Sozialwissenschaftlerin, Externe Psychiatrische Dienste des Kantons Basel-Landschaft

b Dr. med. Dipl. Psych., Oberarzt, Externe Psychiatrische Dienste des Kantons Basel-Landschaft
Korrespondenz: L. Svejda-Hirsch EPD Bruderholz PWS

CH-4101 Bruderholz Tel. 0614254582

lenka.svejda@kpd.ch
Wenn auch nicht auf den ersten Blick ersichtlich, so haben Kultur und Gesundheitspflege doch vieles gemeinsam: Beide repräsentieren Teile von miteinander verwobenen sozialen Realitäten. Menschen, die verschiedenen Kulturkreisen angehören, haben auch entsprechend unterschiedliche inhaltliche Vorstellungen, Erwartungen und praktische Bezüge zu Gesundheit und Krankheit, ebenso wie zu verschiedenen Behandlungsmethoden. Individuen aus unterschiedlichen Herkunftskulturen zeigen im Krankheitsfall oftmals abweichende Verhaltensweisen, die in der Tradition ihrer Sozialisationskultur fussen. Ohne Berücksichtigung dieser Unterschiede bei der medizinischen Behandlung, insbesondere bei chronischen und/oder psychosomatischen Leiden, ist denn auch kaum eine dauerhafte Genesung zu erzielen.

«Fremde» in der Schweiz entstammen vielfach anderen Medizinkulturen. Entsprechend prallen bei Krankheitsfällen und ärztlichen Interventionen verschiedene Welten aufeinander. Es gibt keine Rezeptsammlung für den Umgang mit Menschen aus anderen Kulturen. Doch die Beziehungen, die im Zentrum einer jeden medizinischen Interaktion zwischen $\mathrm{Pa}$ tient, Patientin und Fachperson stehen, können bewusst und unter der Berücksichtigung der bekannten Aspekte gestaltet werden. Die Sensibilisierung hinsichtlich möglicher Verschiedenheiten und Konfliktzonen in der Beratung und Behandlung von Menschen mit Migrationshintergrund kann ein erster Schritt sein. Der zweite ist es dann, nicht nur die Unterschiede und Eigenarten des Anderen zu erfassen, sondern ebenso die Gemeinsamkeiten zu suchen.

Der Aufbau einer vertrauensvollen therapeutischen Beziehung spielt in der Behandlung von Menschen mit Migrationshintergrund eine zentrale Rolle. Patienten aus vorwiegend kollektiv sozialisierenden Kulturen betrachten insbesondere Ärzte als Autoritäten und erwarten, von diesen ihre Symptome «abgenommen» zu bekommen, z. B. durch verordnete Medikamente. Die Grundhaltung solcher Patienten ist in der Regel passiv-rezeptiv. Das wiederum steht der ärztlichen Erwartungshaltung der Ärzte gegenüber, die von ihren Patienten ein gewisses Mass an Selbstverantwortung

\section{Changement de culture} et maladie: différences et points

\section{communs}

Le traitement médical de personnes issues de l'immigration requiert une approche soit globale, soit biologique, psychologique et sociale, dénuée de tout jugement ou préjugé. Le cas échéant, des connaissances de la culture d'origine du patient ou les services d'un interprète/médiateur sont nécessaires pour aboutir à des interactions fructueuses entre le patient et le thérapeute. Un thérapeute disposant de compétences transculturelles affirmées doit être prêt à s'immerger dans un monde différent et à en appréhender les divergences de perspectives. C'est le seul moyen de créer une relation thérapeutique fructueuse dans un environnement transculturel. L'aptitude à aborder l'autre sans préjugés face à sa situation personnelle de vie et de maladie représente une des principales compétences transculturelles. L'aspect de la réciprocité, l'idée directrice de l'intégration basée sur les droits et les devoirs doit être abordée au cours du traitement.

als auch eine aktive Mitarbeit im (psycho-)therapeutischen Prozess und einen klar definierten Behandlungsauftrag erwarten. Diese unterschiedlichen Erwartungshaltungen können zu gegenseitigen Enttäuschungen und Verwirrungen führen. Deshalb ist in noch höherem Masse als bei der Behandlung von Schweizer Patientinnen und Patienten eine aktive und auf Psychoedukation ausgerichtete Grundhaltung notwendig - wobei wir hier tendenziell von Patienten aus bildungsfernen und sozioökonomisch tiefstehenden Milieus reden. 


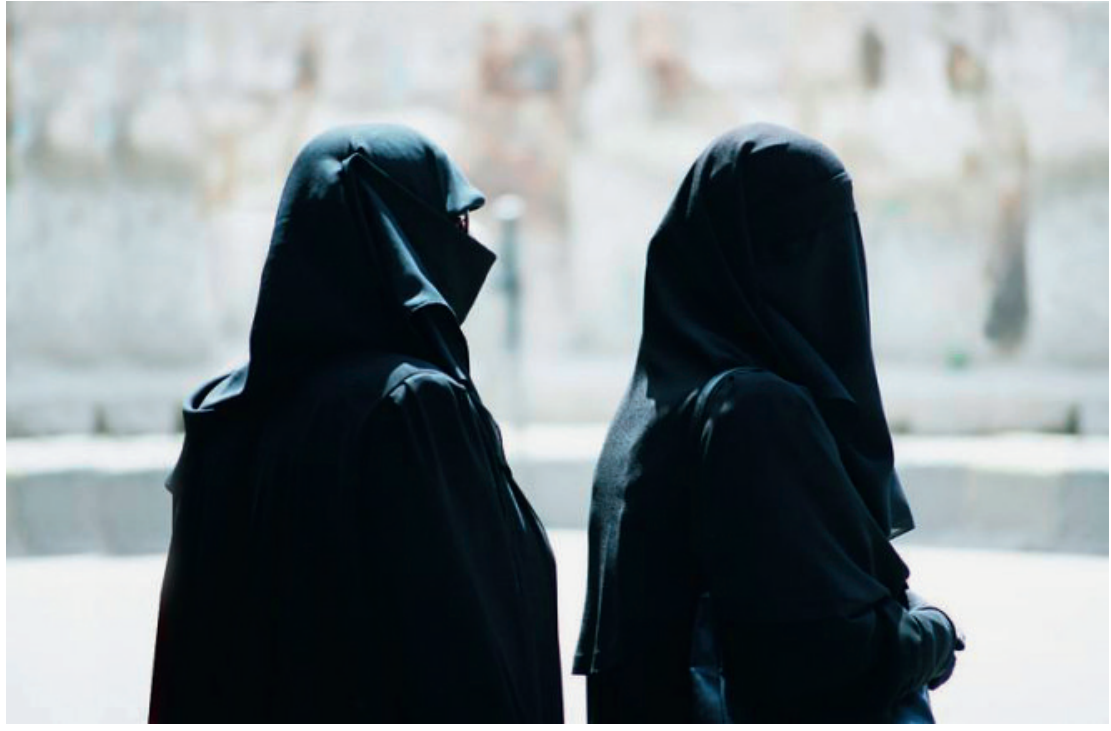

Nicht nur bei Menschen aus weit entfernten Kulturkreisen kann die Beziehung von Arzt und Patient durch Missverständnisse belastet sein.

\section{Exkurs: Kulturbegriff}

Kulturen sind intersubjektive Symbolsysteme, die die Realitäten und die Lebensprozesse kultureller Gemeinschaften strukturieren. Es entstehen Kulturmuster, die repetitiv angewendet werden, im Einzelfall reden wir von Lebenswelten. Die Lebenswelt eines Individuums bezeichnet den Bereich des selbstverständlichen, alltäglichen Wissens sowie der zwischenmenschlichen Erfahrungswelt und aller zugehörigen Primärerfahrungen, einschliesslich des Phänomens «Krankheit». dessen, im 21. Jahrhundert, heisst der politisch korrekte Begriff «Transkulturalität» oder auch «Diversity Management».

Zentral im Raum steht jedoch immer noch die Frage: Wie hängen die Wahrnehmung von der Wirklichkeit, das Denken und der äussere Ausdruck der inneren Empfindungen zusammen? Prallen in Form zweier Menschen unterschiedlicher Herkunft zwei verschiedene kulturelle Systeme aufeinander, so können Kommunikationsschwierigkeiten auftreten, im Krankheitsfall zwischen Fachperson und Patient. Diese sind meistens umso grösser, je entfernter und unterschiedlicher die Herkunftskulturen der betreffenden Personen sind. Als Beispiel für unterschiedliche normative Grundhaltungen sei das Verhalten von türkischstämmigen Männern genannt, das sehr stark von einem für liberal eingestellte europäische Ärztinnen und Ärzte schwer nachvollziehbaren Verständnis von Ehre geprägt ist. Oft werden mehrere Gespräche benötigt, um die unterschiedlichen, kulturbasierten Normensysteme von Behandelndem und Patient zu klären, dessen Reaktionen zu verstehen und zugleich klar und unmissverständlich das normative System des Gastlandes zu vertreten. Gelingt der Balanceakt nicht, so führen «interkulturelle Missverständnisse» nicht selten zum Abbruch der therapeutischen Beziehung.

Wirksam sind aber auch andere verbindende oder trennende Faktoren wie Bildungsstand, gemeinsame Sprachkenntnisse, die Art des Problems, das Geschlecht der Beteiligten, aktuelle Lebenssituation und dergleichen mehr. Da wir vielschichtig kommunizieren, ist auch nicht die Sprache (oder deren Unkenntnis) allein für Kommunikations- und Interaktionspro-

\section{«Transkulturelle Kompetenz beinhaltet im Wesentlichen die Fähigkeit, anderen Menschen in ihrer individuellen Lebens- und Gesundheits- situation vorurteilsfrei begegnen zu können»}

«Kultur» ist also eine kollektive, stetem Wandel unterworfene, verbindende Realität, deren Bedeutungsgehalte immer wieder neu definiert und ausgehandelt werden (müssen). Ein geteilter kultureller Hintergrund kann eine gemeinsame VerständnisBasis darstellen. Je nach biographischem Werdegang und der aktuellen Lebenswelt eines Individuums können bedeutungsvolle Werte aber unterschiedlich definiert werden. So betrachtet kann «Kultur» keinesfalls als abschliessende Begründung, weder bei der Diagnostik noch in der therapeutischen Beziehungsgestaltung, dienen.

\section{Transkulturelle Konzepte}

In den 80er Jahren wurde von den kulturspezifischen Krankheitsklassifikationen gesprochen, die Gesellschaft wurde kulturspezifisch wahrgenommen. Unter- bleme verantwortlich zu machen. Die Sprache an sich ist nur ein Symbolträger für Zeichen und deren Bedeutungen, die weit unter der verbalen Oberfläche liegen. Gerade in der Psychiatrie und Psychosomatik, wo an den unsichtbaren Schnittstellen von «Körper und Seele» gearbeitet wird, ist auf «ungehörte Botschaften» von körperlichen Symptomen zu achten. Diese müssen rechtzeitig wahrgenommen werden, und es muss versucht werden, ihre Bedeutung für das psychische Erleben zu verstehen.

Während psychosomatische Zusammenhänge für Angehörige mitteleuropäischer Kulturen in der Regel leichter zu vermitteln sind, wird seelisches Leiden in anderen («somatisierenden») Kulturkreisen als sehr schambesetzt erlebt, so dass eine vorsichtige «Übersetzungsarbeit» geleistet werden muss. Problematisch dabei ist, dass oftmals die psychische Grundlage der 
somatoformen Symptome über viele Jahre nicht erkannt wurde, und diese Patienten jahrelang von Arzt zu Arzt, von Spital zu Spital gewandert sind und die Symptome unterdessen entsprechend chronifiziert sind. Der Ausbildung und Beratung von Primärärzten im Umgang mit Patienten aus anderen Kulturen sollte daher eine besondere Aufmerksamkeit zuteil werden.

\section{Migration oder: \\ Macht Auswanderung krank?}

Im Fall einer Migration wird nicht nur das gewohnte Lebensumfeld verlassen, sondern auch das bekannte und vertraute Medizinsystem. Das Individuum erfährt insbesondere bei einer unfreiwilligen Migration Existenzverunsicherungen, Trennungen mit Verlust emotionaler Bindungen und sozialer Netze, eventuell Bedrohungen, trifft auf unbekannte soziale Realitäten und Kulturmuster usw. Hinzu kommt, dass dieses Erleben chronologisch nicht gestaffelt, sondern oft synchron geschieht, d. h. alles gleichzeitig einbricht, und kumuliert zu einer enormen psychischen Belastung wird. Der Migrations- und Integrationsprozess kann, je nach erlebten Belastungen, kürzer sein oder auch jahrelang andauern, ggf. auch nie zum Abschluss kommen. Kommt ein unsicherer Aufenhaltsstatus und damit die Ungewissheit bezüglich der näheren Zukunft hinzu, so sind oft Ängste, Depressionen oder auch paranoide Reaktionen die Folge der als Stressoren wirkenden, ungünstigen Lebensumstände. Kumulieren sich diese Faktoren, so erhöht sich die Vulnerabilität und psychische und psychosomatische Störungen können resultieren. Soziale Problemlagen und persönliche Ressourcen haben einen grossen Einfluss auf die psychische, emotionale und physische Gesundheit - und das nicht nur bei Personen mit Migrationshintergrund. Bei letzteren kann z. B. eine Migrationsanamnese Sinn machen und notwendige Erklärungen liefern.

\section{Alltagspraxis und transkulturelle Kompetenz}

Die Forderung nach Chancengleichheit im Gesundheitssystem (BAG) birgt nicht zuletzt ein ökonomisches Interesse in sich: Fühlen sich ausländische Patienten missverstanden oder «schlecht behandelt», resultiert ein «Ärzte-(S)Hopping», das in der Regel weder den Patienten nachhaltig hilft noch der Finanzsituation im Gesundheitswesen gut tut. So gehört es z. B. zu kulturspezifischen Erfahrungshorizonten, intrafamiliäre Konflikte indirekt durch Krankheiten zu thematisieren: Die Somatisation psychischen Leidens ist sehr häufig. Ein weiterer Themenkreis ist die Sozialisation: Vielfach steht unser Individualismus einer kollektiv orientierten Sozialisation gegenüber, die ein- schliesst, dass über persönliche Gefühle nicht gesprochen wird.

Aus all diesen Gründen ist es notwendig, in der Behandlung von Menschen mit Migrationshintergrund einen möglichst ganzheitlichen oder bio-psycho-sozialen und in der Begegnung selbst wertfreien Ansatz zu praktizieren. Gegebenenfalls braucht es Kenntnisse der Herkunftskultur oder Dolmetscher-/Mediatorendienste für erfolgreiche Therapeuten-Patienten-Interaktionen. Ein/e transkulturell versierte/r Therapeutin bzw. Therapeut muss die Bereitschaft haben, sich auf die eventuell fremden Lebenswelten einzulassen, den Versuch einer Perspektivenübernahme einzugehen und die Bereitschaft zur Empathie mitbringen. Nur so kann ein transkulturell erfolgreiches therapeutisches Setting initiiert werden. Der Aspekt der Gegenseitigkeit, des Integrationsleitgedankens «fördern und fordern» ist im Laufe der Behandlung zu thematisieren.

Transkulturelle Kompetenz beinhaltet im Wesentlichen die Fähigkeit, anderen Menschen in ihrer individuellen Lebens- und Gesundheitssituation vorurteilsfrei begegnen zu können. Sie ist im Gesundheitswesen von grosser Wichtigkeit, denn unreflektierte Verallgemeinerungen und Vorurteile verhindern den Blick auf die tatsächlichen Probleme von Patienten und damit auch eine angemessene Behandlung.

Beziehungen aller Art, also auch therapeutische, bilden ein zentrales Muster, das zusammen mit der Wahrnehmung die Lebenswelt des Einzelnen weitgehend bestimmt. Ein möglichst gegenseitiges, kulturelles Bezugswissen und die Berücksichtigung der individuellen Lebenswelten der Patienten können eine für beide Seiten positive Interaktion ermöglichen. Nachfolgend ein der Patientenzufriedenheitsbefragung 2008 entnommenes Zitat: «Da ich Deutsche bin und Ausländer[in], bin ich sehr froh, dass es bei Ihnen auch deutsche Ärzte, Psychologen gibt, da ich [mich] von denen besser verstanden fühle, also es keine Sprachbarrieren gibt.»

Den Fragen nach Sinnhaftigkeit, lebensweltlicher Orientierung und ähnlichem nachzugehen und das patienteneigene Erklärungsmodell zu entwickeln, funktioniert am besten im Kontext einer vertrauensvollen Beziehung. Ein respektvoller Umgang mit den Patienten kann z.B. in der Einstiegsfrage «Ich weiss, verschiedene Menschen haben unterschiedliche Vorstellungen von Krankheit ... bitte helfen Sie uns, Ihre Sicht der Dinge zu verstehen» zum Ausdruck gebracht werden. Und wie das Patientenzitat zeigt, ist auch kein «Exotismus» notwendig, manchmal fangen «fremde Welten» bereits vor der Haustüre an. 